\title{
A COMPLEXIDADE DO QUADRANTE PÓSTERO-LATERAL NAS FRATURAS DO PLANALTO TIBIAL: RELATO DE UM CASO ATÍPICO E REVISÃO DA LITERATURA
}

\author{
THE COMPLEXITY OF THE POSTEROLATERAL QUADRANT IN FRACTURES OF THE \\ TIBIAL PLATEAU: REPORT OF AN ATYPICAL CASE AND REVIEW OF THE LITERATURE
}

Robinson Esteves Pires,TCBC-PA ${ }^{1,2}$; Vincenzo Giordano, TCBC-RJ3; Mauricio Kfuri ${ }^{4}$; Fabricio Fogagnolo ${ }^{5}$; Gustavo Waldolato ${ }^{6}$; Frederico Pimenta ${ }^{1}$; Tulio Vinícius Oliveira Campos ${ }^{1}$; Marco Antônio Percope de Andrade ${ }^{1}$.

\section{RESUMO}

Determinados padrões de fratura do planalto tibial podem representar um quadro atípico de luxação ou subluxação do joelho, cuja suspeição diagnóstica pode não ser óbvia na avaliação inicial, especialmente se o joelho estiver reduzido nos exames de imagem. A associação entre a fratura pósterolateral do planalto tibial e as lesões do ligamento cruzado anterior, do ligamento colateral medial e do menisco lateral é relativamente frequente. Alguns pacientes podem apresentar um padrão raro de depressão articular na região póstero-lateral do planalto tibial, em associação com a lesão do ligamento cruzado anterior, que se assemelha à mordida de maçã, razão pela qual foi denominado "apple-bite-fracture". O objetivo do presente estudo é descrever um quadro atípico de luxação de joelho, acompanhado por um padrão ainda mais raro de fratura, onde há destacamento de um fragmento osteocondral por forças de cisalhamento, entendido como uma variação do padrão "apple-bite", e seu tratamento com a realização da osteotomia do epicôndilo lateral do fêmur. Descritores: Tíbia. Fraturas da Tíbia. Joelho. Traumatismos do Joelho.

\section{ABSTRACT}

Certain fracture patterns of the tibial plateau may represent an atypical knee dislocation or subluxation, whose diagnosis may not be obvious in the initial assessment, especially if the knee is reduced in imaging studies. The association between posterolateral fracture of the tibial plateau and injuries of the anterior cruciate ligament, medial collateral ligament, and lateral meniscus is relatively frequent. Some patients may present a rare pattern of joint depression in the posterolateral region of the tibial plateau, in association with the injury of the anterior cruciate ligament, which resembles an apple bite, which is why it was called "apple-bite-fracture". The aim of the present study is to describe an atypical knee dislocation, accompanied by an even rarer fracture pattern, in which an osteochondral fragment is detached by shear forces, understood as a variation of the "apple-bite" pattern, and treatment with osteotomy of the lateral femoral epicondyle. Keywords: Tibia. Tibial Fractures. Knee. Knee Injuries.

\section{INTRODUÇÃO}

As fraturas da região posterior do planalto tibial são relativamente frequentes e de dificil tratamento. Xiang et al. ${ }^{1}$, em série de 242 pacientes com fraturas do planalto tibial, reportaram que $15 \%$ acometeram a região póstero-lateral do joelho. Zhu et al. ${ }^{2}$ relataram uma incidência ainda maior $(44.32 \%)$ de acometimento da região póstero-lateral do planalto tibial nas fraturas bicondilares. O correto entendimento da personalidade da fratura do planalto tibial é de fundamental importância na tomada de decisão sobre a escolha da via de acesso e

\footnotetext{
${ }^{1}$ Universidade Federal de Minas Gerais, Departamento do Aparelho Locomotor - Belo Horizonte, MG, Brasil ${ }^{2}$ Hospital Felicio Rocho, Serviço de Ortopedia e Traumatologia - Belo Horizonte, MG, Brasil

${ }^{3}$ Hospital Municipal Miguel Couto, Serviço de Ortopedia e Traumatologia Professor Nova Monteiro - Rio de Janeiro, RJ, Brasil

4University of Missouri, Missouri Orthopaedic Institute - Columbia - Missouri - Estados Unidos

5Hospital das Clinicas Da Universidade de São Paulo, Departamento de Ortopedia e Anestesiologia - Ribeirão Preto - SP - Brasil

${ }^{6}$ Faculdade de Ciências Médicas de Minas Gerais, Hospital Universitário, Serviço de Ortopedia e Traumatologia Belo Horizonte - MG - Brasil
} 
Pires et al.

A complexidade do quadrante póstero-lateral nas fraturas do planalto tibial: relato de um caso atípico e revisão da literatura.

Relato de caso

da estratégia de fixação da fratura. Diversos autores propuseram novos sistemas de classificações abrangendo os elementos posteriores do planalto tibial, uma vez que a classificação original de Schatzker, apesar de universalmente utilizada, identifica o padrão (cisalhamento e/ou afundamento) e a localização do traço de fratura somente no plano ântero-posterior ${ }^{3-6}$. Trata-se, portanto, de um sistema bidimensional. Recentemente, Kfuri e Schatzker ${ }^{7}$ revisitaram a classificação original do último autor e propuseram uma adaptação baseada na interpretação tomográfica e tridimensional da fratura do planalto tibial. Segundo os autores, o planalto tibial é dividido em duas colunas (lateral e medial). Um equador virtual divide o planalto tibial, no corte tomográfico axial, em quadrantes anterior e posterior. O equador possui como limites o tubérculo lateral da fíbula (inserção do ligamento colateral lateral) e a crista posterior da tíbia (inserção do ligamento colateral medial). Uma vez determinada a localização do plano principal da fratura, entende-se o mecanismo do trauma e as possiveis lesões associadas, para que possa ser definida estratégia de tratamento.

No entanto, determinados padrões de fratura póstero-lateral do planalto tibial podem denotar uma apresentação atípica de luxação ou subluxação do joelho. Portanto, avaliação pormenorizada da articulação deve ser realizada, tendo-se em conta que a fratura póstero-lateral do planalto tibial pode estar associada a lesões menisco-ligamentares.

Kaplan et al. 8 e Stallenberg et al. 9 descreveram a associação entre a lesão do ligamento cruzado anterior e a fratura oculta do quadrante póstero-lateral do planalto tibial. A ruptura do ligamento cruzado anterior, sob estresse em valgo, leva à translação anterior e à rotação interna da tíbia em relação ao fêmur. Ocorre, então, a impacção da porção posterior do planalto tibial lateral contra o meio do côndilo femoral lateral, ocasionando uma zona de edema ósseo no quadrante póstero-lateral do planalto tibial, com ou sem edema ósseo associado no côndilo femoral lateral ("kissing contusion / bone bruise"). Menzdorf et al.10 reportaram que o impacto do côndilo femoral sobre o planalto tibial, secundário à subluxação anterior da tíbia, pode determinar um padrão específico de impacção na margem póstero-lateral do planalto que se assemelha à "mordida de maçã", razão pela qual recebeu a denominação de "apple-bitefracture".

Andrade et al. ${ }^{11}$ descreveram um padrão de fratura osteocondral desviada póstero-lateral do planalto tibial associado à lesão do ligamento cruzado anterior que difere da impacção clássica descrita como "apple-bite". A fratura em questão apresentava fragmento osteocondral grande e completamente desviado (90\%), sugerindo um mecanismo de entorse e subluxação anterior do joelho que, no momento da redução, levou o fragmento para o compartimento anterior do mesmo.

Os objetivos do presente estudo são descrever um quadro atípico de subluxação do joelho representado pela associação de um padrão raro de fratura póstero-lateral do planalto tibial, aqui denominado "applebite" variante, com lesão do ligamento cruzado anterior e do menisco lateral, assim como apresentar uma análise crítica da literatura.

\section{RELATO DO CASO}

Paciente do gênero feminino, 49 anos, sofreu queda da bicicleta e apresentou dor, impotência funcional e impossibilidade de marcha. Os exames de imagem evidenciaram fratura póstero-lateral do planalto tibial esquerdo (Figura 1). Devido ao derrame articular importante e ao desvio do fragmento osteocondral fraturado, que se encontrava em posição subcutânea e inclinado $90^{\circ}$ em relação à superficie articular, a possibilidade de lesão ligamentar do pivô central foi levantada, mas não confirmada, devido à indisponibilidade da ressonância magnética.
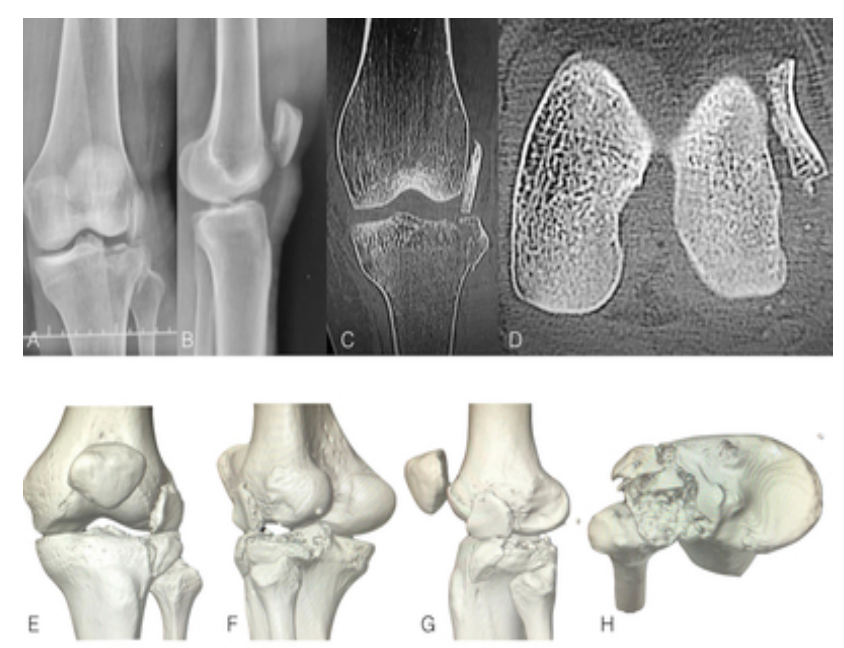
Pires et al.

A complexidade do quadrante póstero-lateral nas fraturas do planalto tibial: relato de um caso atípico e revisão da literatura.

Relato de caso

Figura 1. Radiografias do joelho esquerdo em ânteroposterior e lateral (A e B) mostrando a fratura póstero-lateral do panalto tibial. Cortes tomográficos coronal (C) e axial (D) mostrando o fragmento osteocondral com desvio de 90 graus em relação à superficie articular. Reconstrução tridimensional da tomografia computadorizada demonstrando a fratura e o desvio do fragmento osteocondral (E, F, G, H).

A estratégia de tratamento foi a osteossintese da fratura e possivel reparo da lesão meniscal, deixando-se a lesão ligamentar para segundo tempo, caso houvesse instabilidade tardia no joelho, uma vez que a paciente não praticava esportes de contato com movimentos rotacionais ou mudanças súbitas de direção.

\section{Procedimento cirúrgico}

Com a paciente posicionada em decúbito lateral direito e torniquete colocado na raiz do membro inferior esquerdo, a via de acesso realizada foi a ântero-lateral estendida, com $10 \mathrm{~cm}$ de comprimento, inclinando-se proximal ao epicôndilo lateral do fêmur e com término entre a tuberosidade da tíbia e a cabeça da fibula (Figura 2A). Após a dissecção do tecido celular subcutâneo, foi possivel identificar o fragmento osteocondral fraturado, situado anteriormente ao trato iliotibial. A cápsula articular lateral e parte do trato iliotibial foram rompidos pelo próprio fragmento fraturado ( $\mathrm{Fi}$ gura 2B). O epicôndilo lateral do fêmur foi identificado e um fio de Kirschner de $1,5 \mathrm{~mm}$ foi posicionado no seu centro, de lateral para medial, para certificação da correta localização do sítio da osteotomia, sob visão radioscópica. Procedeu-se, então, à osteotomia do epicôndilo lateral do fêmur e o mesmo foi rebatido posteriormente, junto com o tendão popliteo e o ligamento colateral lateral, conforme descrito por Bowers et al. ${ }^{12}$ e Kfuri et al. ${ }^{13}$. Parafusos de Schanz colocados no fêmur e na tíbia foram conectados por uma barra lateral de fixador externo tubo-a-tubo, com a finalidade de promover abertura do compartimento lateral do joelho, possibilitando melhor visualização da superficie articular (Figura 2C). Com a abertura do espaço articular lateral, foi possivel a identificação das lesões do ligamento cruzado anterior e do menisco lateral. Procedeu-se então à redução e fixação provisória da fratura com fios de Kirschner (Figura 2D). Após confirmação da obtenção de redução anatômica, sob visão radioscópica, a fratura foi fixada com um parafuso Herbert, dois parafusos corticais $2,4 \mathrm{~mm}$ (um de superior para inferior e outro de anterior para posterior) e um parafuso esponjoso $3,5 \mathrm{~mm}$, com rosca parcial, inserido na periferia do fragmento fraturado. Uma placa de $2,4 \mathrm{~mm}$ foi adicionada para suportar o cisalhamento anterolateral (Figura 2E). A lesão radial do menisco lateral foi suturada (Figura 3A) e a osteotomia do epicôndilo lateral foi fixada com parafuso cortical 4,5mm e arruela denteada (Figura 3B). As imagens intraoperatórias ilustram a redução e a fixação da fratura (Figuras $3 \mathrm{C}$ e $3 \mathrm{D}$ ). Foi optado pelo tratamento não cirúrgico da lesão do ligamento cruzado anterior neste tempo pelas razões explicitadas anteriormente.
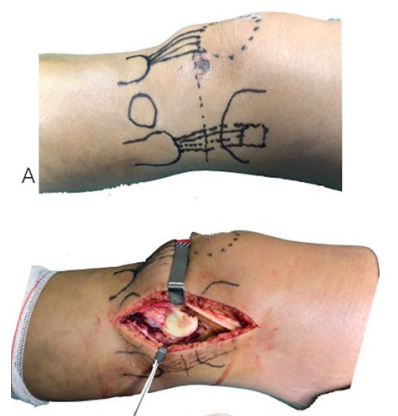

D
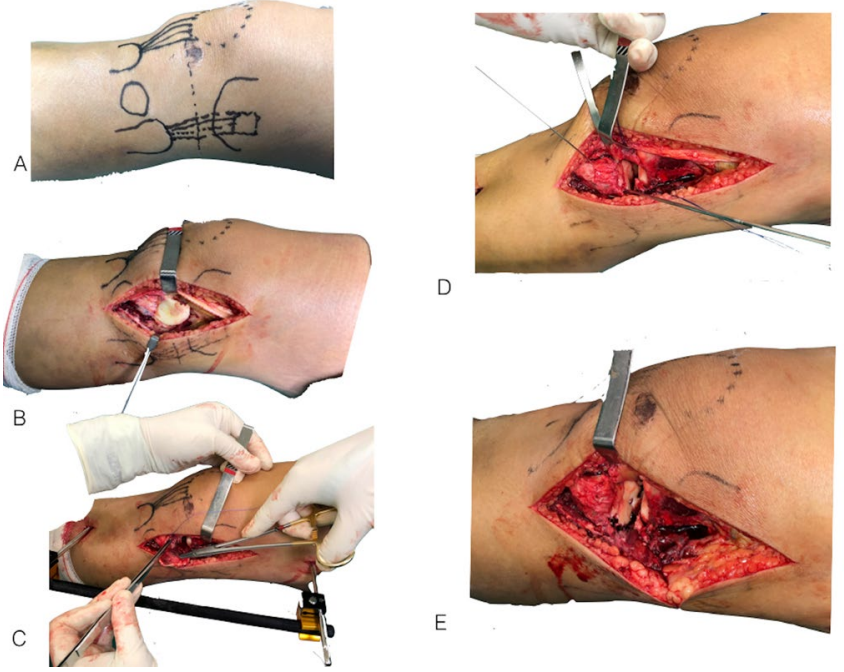

Figura 2. Fotografia pré-operatória mostrando os pontos de reparo para a realização da via anterolateral estendida (tuberosidade da tíbia, ligamento colateral lateral e epicôndilo lateral do fêmur) (A). Fotografia intraoperatória mostrando o fragmento osteocondral desviado (B). Fotografia intraoperatória mostrando a abertura do espaço articular lateral com a colocação do fixador externo lateral (C). Redução da fratura e fixação provisória com fios de Kirschner (D). Aspecto da superficie articular do planalto tibial após a redução e fixação da fratura com parafusos $(\mathrm{E})$.
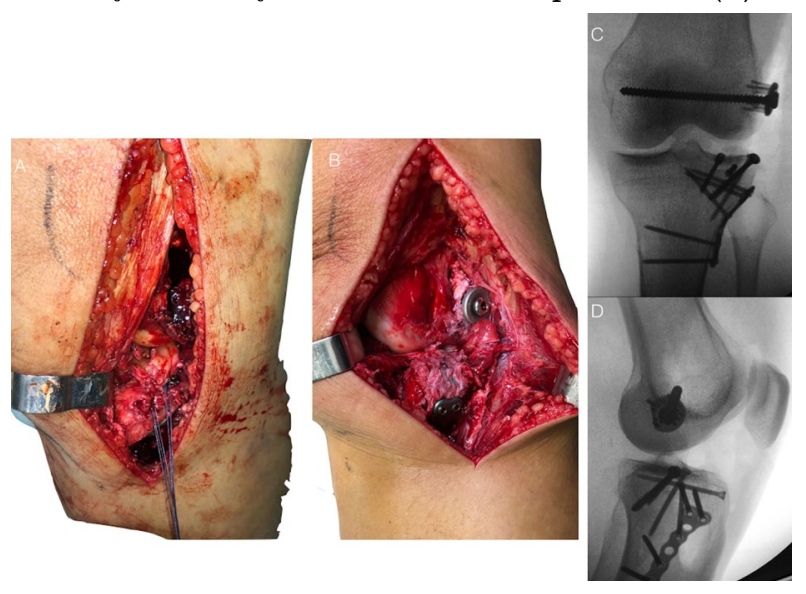
Pires et al.

A complexidade do quadrante póstero-lateral nas fraturas do planalto tibial: relato de um caso atípico e revisão da literatura.

Relato de caso

Figura 3. Fotografias intraoperatórias mostrando a sutura da lesão radial do menisco (A) e a fixação da osteotomia do epicôndilo lateral (B). Imagens radioscópicas pós-operatórias em ântero-posterior (C) e perfil (D) mostrando a redução e fixação da fratura.

\section{Protocolo pós-operatório}

A paciente recebeu analgesia endovenosa e profilaxia antibiótica por 24 horas. A alta hospitalar ocorreu no segundo dia. Mobilização ativa do joelho, sem restrição de amplitude de movimento, e exercícios isométricos foram permitidos a partir do segundo dia de pós-operatório, conforme tolerância. Carga parcial proprioceptiva, com auxílio de duas muletas, foi permitida a partir da segunda semana e mantida por mais sete semanas. Após oito semanas, a paciente permaneceu com carga parcial (uma muleta contralateral) até a $12^{\mathrm{a}}$ semana. Após este periodo, carga total foi liberada e o fortalecimento muscular foi progressivamente aumentado.

Após 12 semanas, a fratura estava completamente consolidada (Figuras 4A e 4B) e a paciente apresentava amplitude de movimento de $0-110^{\circ}$ (Figura 4C e 4D) e discreta claudicação.

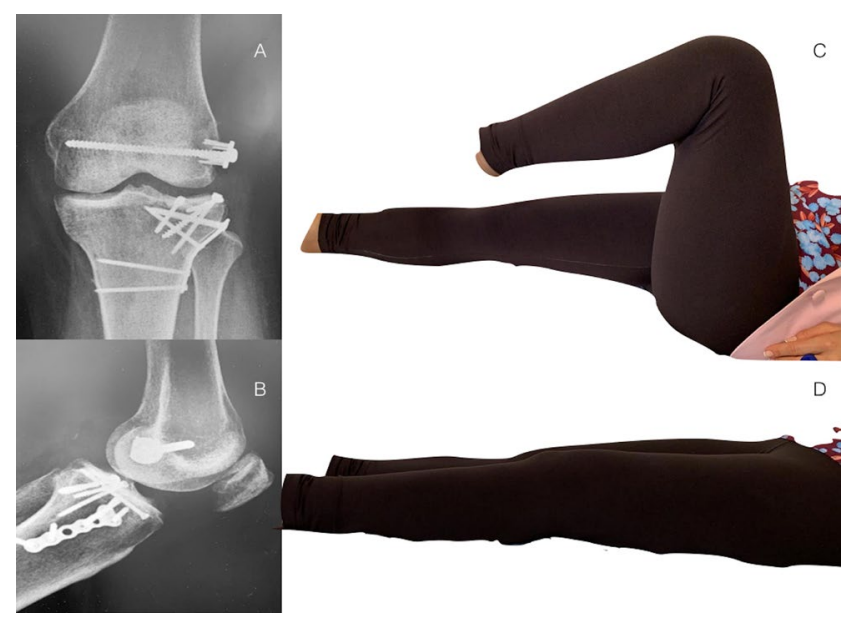

Figura 4. Radiografias do joelho esquerdo em ânteroposterior (A) e perfil (B) mostrando consolidação da fratura após 12 semanas da osteossintese. Observe a amplitude de movimento do joelho de $0-110^{\circ}$ (C e D).

Após um ano, a paciente apresentava-se completamente sem dor e retornou às suas atividades laborais e físicas no mesmo nivel funcional prévio à lesão. A figura 5 demonstra a consolidação da fratura (Figuras $5 \mathrm{~A}$ e $5 \mathrm{~B})$. Observe a amplitude de movimento completa do joelho operado (Figuras $5 \mathrm{C}$ e $5 \mathrm{D})$. A pontuação pelo escore de Lysholm, validado para a lingua portugue$\mathrm{sa}^{14}$, foi de 95 pontos, traduzindo um resul- tado funcional considerado excelente. Segundo o critério radiográfico de Rasmussen modificado ${ }^{15}$, a paciente apresentou pontuação 10, o que também denota excelente resultado radiográfico.
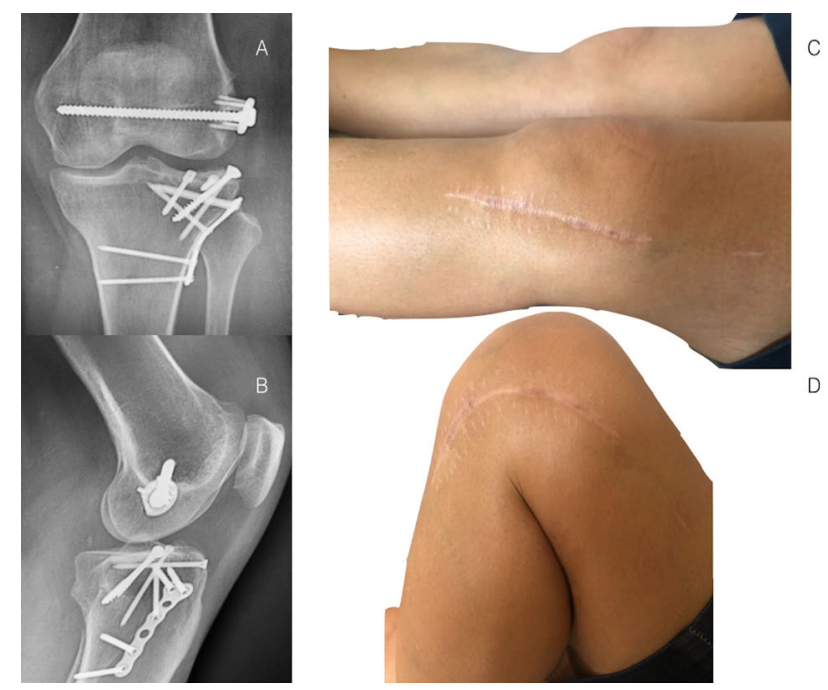

Figura 5. Radiografias do joelho esquerdo em ânteroposterior (A) e perfil (B), após 1 ano de seguimento, evidenciando consolidação da fratura e ausência de sinais de osteoartrite. Observe a amplitude de movimento completa do joelho operado.

\section{Consentimento informado}

Consentimento informado foi obtido e se encontra devidamente assinado e disponivel para avaliação. Aprovação ética pelo CEP (CAAE: 36469720.0.0000.5125).

\section{DISCUSSÃo}

O objetivo do tratamento da fratura do planalto tibial é a redução anatômica, com restauração do eixo e fixação estável. Entretanto, atualmente não existe consenso sobre quando uma fratura póstero-lateral do planalto tibial deva ser reparada, uma vez que essa região representa, em média, $22,6 \mathrm{~mm}$ do total da superficie articular do planalto tibial ${ }^{16}$. Diversos autores têm demonstrado o grau de depressão articular como preditor de lesões de tecidos moles na fratura do planalto tibial, levando potencialmente à translação dinâmica anterior da tíbia, com subsequente sobrecarga e instabilidade rotatória póstero-lateral 17-19. Chang et al. ${ }^{17}$ encontraram um risco maior de lesão do menisco lateral com mais de $6,3 \mathrm{~mm}$ de depressão da articulação lateral e de ruptura do ligamento cruzado anterior quando a depressão volumétrica da articulação lateral é menor que $209,5 \mathrm{~mm}^{2}$ e/ou há mais de $5,7 \mathrm{~mm}$ de alargamento da articulação lateral. 
Pires et al.

A complexidade do quadrante póstero-lateral nas fraturas do planalto tibial: relato de um caso atípico e revisão da literatura.

Relato de caso

Bernholt et al.20 propuseram um novo sistema de classificação morfológica para as fraturas póstero-laterais do planalto tibial associadas à lesão do ligamento cruzado anterior, baseado na análise de 825 joelhos. Apesar da casuística expressiva, os autores não fizeram menção ao padrão variante "apple-bite" em sua série, o que denota a raridade deste padrão de fratura. Os autores encontraram algum grau de impacção póstero-lateral em 49,3\% dos casos e dividiram as fraturas em três tipos: fraturas da cortical posterior, sem envolvimento articular (I); impacção articular posterior (II); fragmento osteocondral desviado (IIIA: cisalhamento, IIIB: depressão articular). O principal achado deste estudo foi que as variantes do tipo III foram associadas a um aumento da incidência de lesões meniscais laterais, na vigência de lesão concomitante do ligamento cruzado anterior. Além disso, quando uma ruptura do ligamento colateral medial de grau III é observada no exame clínico, deve haver suspeita aumentada (chance cinco vezes maior) de fratura por impacção do planalto tibial póstero-lateral (tipo IIIA) na ressonância magnética.

Dessa forma, nos parece que a restauração sagital anatômica tanto da superficie articular quanto da inclinação tibial posterior é obrigatória para uma amplitude de movimento estável e indolor do joelho. Além disso, é necessária a utilização de um método estável de fixação para manter a redução de forma anatômica e permitir reabilitação imediata.

Atualmente, não há diretrizes sobre a tática cirúrgica ideal para essas lesões. No entanto, as fraturas que acometem a zona posterior da coluna lateral do planalto tibial possuem um obstáculo anatômico, a cabeça da fibula, que dificulta sua redução e fixação. A proximidade das estruturas neurovasculares nobres torna as abordagens posteriores ao planalto póstero-lateral desafiadoras e com risco de lesões iatrogênicas potencialmente catastróficas. Nas últimas décadas, diversos autores descreveram vias de acesso para abordagem das fraturas póstero-laterais do planalto tibial, cada uma com suas vantagens e limitações (Tabela 1) 12,13,21-28.

Neste caso, optou-se pela realização da osteotomia do epicôndilo lateral do fêmur por se tratar de uma abordagem que permite visualização adequada de toda a coluna lateral do planalto tibial. Como não houve cisalhamento posterior no planalto, cuja presença seria uma limitação para a via, a osteotomia do epicôndilo possibilitou redução anatômica e fixação estável da fratura, assim como a sutura da lesão meniscal.

Andrade et al.11 optaram pela osteotomia transfibular para o tratamento de fratura semelhante, também associada à lesão do ligamento cruzado anterior, e obtiveram consolidação anatômica da fratura e resultado funcional favorável após 6 meses de seguimento. Assim como no presente estudo, os autores optaram pela não reconstrução do ligamento cruzado anterior no primeiro tempo.

A reconstrução ou não do ligamento cruzado anterior no primeiro tempo cirúrgico é tema controverso na literatura. Shekhar et al. ${ }^{29}$, recentemente, reportaram três casos de fraturas osteocondrais da tíbia, com lesões associadas do ligamento cruzado anterior, do ligamento colateral medial e da raíz do menisco lateral. Além da fixação da fratura, os autores advogam reparo transtibial para a lesão da raíz meniscal, além de reparo ou reconstrução dos ligamentos cruzado anterior e colateral medial.

No entanto, apesar de agregar estabilidade, a reconstrução aguda do ligamento cruzado anterior aumenta a morbidade operatória e, consequentemente, o risco de complicações. No presente caso, foi optado pelo tratamento não operatório da lesão do cruzado anterior, hipotetizando-se que o processo de cicatrização local ("healing response") poderia determinar estabilidade secundária ao joelho ou que uma possivel instabilidade poderia não ter repercussões funcionais, considerando-se o perfil esportivo da paciente.

A avaliação funcional, realizada após um ano de trauma, demonstrou que a paciente apresentava joelho estável, uma vez que sua pontuação na escala de Lysholm foi 95 pontos, o que é considerado excelente resultado funcional.

Outro ponto a ser destacado é que a avaliação artroscópica tardia poderia trazer informações valiosas do real do estado da cartilagem no planalto tibial e da cicatrização da lesão do menisco lateral, que foi suturada. No entanto, como a paciente encontrava-se completamente assintomática após 
Pires et al.

A complexidade do quadrante póstero-lateral nas fraturas do planalto tibial: relato de um caso atípico e revisão da literatura.

Relato de caso

um ano do procedimento, presume-se que, se houvesse dano articular importante, não consolidação, necrose do fragmento osteo- condral ou não cicatrização da lesão meniscal, o desfecho funcional seria completamente diferente do encontrado.

Tabela 1. Vias de acesso para o tratamento das fraturas póstero-laterais do planalto tibial, com suas vantagens e potenciais limitações

\begin{tabular}{|c|c|c|}
\hline VIA DE ACESSO & VANTAGENS & LIMITAÇÕES \\
\hline $\begin{array}{c}\text { Carlson }^{21} \\
\text { Póstero-lateral }\end{array}$ & $\begin{array}{l}\text { Via direta, sem osteotomias. } \\
\text { Possibilidade de redução de afunda- } \\
\text { mento e cisalhamento póstero- } \\
\text { laterais, com colocação de placa de } \\
\text { suporte. }\end{array}$ & $\begin{array}{l}\text { Janela póstero-lateral limitada. Não permite } \\
\text { acesso à região anterolateral do planalto } \\
\text { tibial. Necessidade de identificação do nervo } \\
\text { fibular. }\end{array}$ \\
\hline $\begin{array}{l}\text { Frosch et al. }{ }^{22} \\
\text { (Lateral estendida com artro- } \\
\text { tomias anterolateral e póstero- } \\
\text { lateral) }\end{array}$ & $\begin{array}{l}\text { Via única, sem osteotomias, para } \\
\text { tratar fraturas da coluna lateral (qua- } \\
\text { drantes anterior e posterior). }\end{array}$ & $\begin{array}{l}\text { Dificuldade de redução do fragmento póste- } \\
\text { ro-lateral, especialmente se houver afunda- } \\
\text { mento articular. Necessidade de identificação } \\
\text { do nervo fibular. }\end{array}$ \\
\hline $\begin{array}{l}\text { Qiu et al. }{ }^{23} \\
\text { "L invertido" }\end{array}$ & $\begin{array}{l}\text { Via única que permite tratar fraturas } \\
\text { posteriores das colunas medial e late- } \\
\text { ral, sem necessidade de abordagem } \\
\text { do nervo fibular. }\end{array}$ & $\begin{array}{l}\text { Acesso limitado à zona posterior da coluna } \\
\text { lateral pela tensão dos gastrocnêmios. Difícil } \\
\text { redução do afundamento articular póstero- } \\
\text { lateral. }\end{array}$ \\
\hline $\begin{array}{c}\text { Chow et al. }{ }^{24} \\
\text { (Anterolateral modificada: } \\
\text { espaço "para-colateral lateral") }\end{array}$ & $\begin{array}{l}\text { Via única que permite a redução e } \\
\text { fixação da fratura dos quadrantes } \\
\text { anterior e posterior da coluna lateral. }\end{array}$ & $\begin{array}{l}\text { Não permite a colocação de placa de suporte } \\
\text { póstero-lateral. É possível somente a coloca- } \\
\text { ção da placa tipo "rim" para a contenção do } \\
\text { fragmento póstero-lateral. }\end{array}$ \\
\hline $\begin{array}{l}\text { "Jail technique" } 25 \\
\text { Sob redução artroscópica }\end{array}$ & $\begin{array}{c}\text { Procedimento minimamente invasivo. } \\
\text { Fixação somente com parafusos. Sem } \\
\text { necessidade de abordagem do nervo } \\
\text { fibular. }\end{array}$ & $\begin{array}{l}\text { Não permite a colocação de placa de suporte } \\
\text { posterior. }\end{array}$ \\
\hline $\begin{array}{l}\text { Lobenhoffer }^{26} / \text { Solomon }^{27,28} \\
\text { (Osteotomia transfibular) }\end{array}$ & $\begin{array}{l}\text { Visualização adequada de toda a co- } \\
\text { luna lateral (quadrantes anterior e } \\
\text { posterior). Permite colocação de pla- } \\
\text { cas de suporte anterolateral e póste- } \\
\text { ro-lateral, assim como placas horizon- } \\
\text { tais tipo "rim" ou "hoop". }\end{array}$ & $\begin{array}{l}\text { Dano à articulação tibiofibular proximal. Ne- } \\
\text { cessidade de abordagem do nervo fibular. } \\
\text { Necessidade de fixação da fíbula. }\end{array}$ \\
\hline $\begin{array}{l}\text { Bowers }^{12} / \text { Kfuri et al. } \\
\text { (Osteotomia do epicôndilo } \\
\text { lateral do fêmur) }\end{array}$ & $\begin{array}{l}\text { Excelente visualização de toda a colu- } \\
\text { na lateral do planalto tibial. Sem ne- } \\
\text { cessidade de identificação e isolamen- } \\
\text { to do nervo fibular. Permite a fixação } \\
\text { de fragmentos osteocondrais. Permite } \\
\text { a fixação de fratura-avulsão do liga- } \\
\text { mento cruzado anterior. Acesso am- } \\
\text { plo para sutura meniscal. }\end{array}$ & $\begin{array}{l}\text { Não permite a colocação de placa de suporte } \\
\text { póstero-lateral. Necessidade de fixação do } \\
\text { epicôndilo após a fixação do planalto. }\end{array}$ \\
\hline
\end{tabular}


O presente estudo apresenta algumas limitações que devem ser pontuadas: não foi realizada ressonância magnética pré-operatória, exame importante para a confirmação das lesões meniscal e ligamentar na vigência de um padrão não usual de fratura, devido à sua indisponibilidade; o seguimento pós-operatório de um ano, apesar de mostrar resultado funcional favorável do ponto de vista da confirmação da consolidação da fratura e da potencial cicatrização da lesão meniscal, não exclui o risco de degeneração articular tardia do joelho; e não foi realizada a medida do Lachman com artrômetro (KT 1000), o que certamente agregaria informação mais objetiva sobre a estabilidade do joelho.

\section{CONCLUSÃo}

A fratura póstero-lateral isolada do planalto tibial pode representar a apresentação atípica de uma luxação ou subluxação do joelho. Dependendo da idade do paciente e da demanda funcional, reconstrução ligamentar primária pode estar indicada. A osteotomia do epicôndilo lateral do fêmur possibilita redução adequada e fixação estável para a fratura póstero-lateral tipo "apple-bite" variante.

\section{REFERÊNCIAS}

1. Xiang G, Zhi-Jun P, Qiang Z, Hang L. Morphological characteristics of posterolateral articular fragments in tibial plateau fractures. Orthopedics. 2013;36(10):e1256-61.

2. Zhu Y, Meili S, Dong MJ, Zhai QL, Yao L, Wang JC, et al. Pathoanatomy and incidence of the posterolateral fractures in bicondylar tibial plateau fractures: a clinical computed tomography-based measurement and the associated biomechanical model simulation. Arch Orthop Trauma Surg. 2014;134(10):1369-80.

3. Zhu Y, Yang G, Luo CF, Smith WR, $\mathrm{Hu} \mathrm{CF}$, Gao H, et al. Computed tomography-based Three-Column Classification in tibial plateau fractures: introduction of its utility and assessment of its reproducibility. J Trauma Acute Care Surg. 2012; 73(3):731-7.

4. Chang SM, Hu SJ, Du SC, Ma Z, Xiong WF, Yao XZ. Fourquadrant/column classification of tibial plateau fractures. Int Orthop. 2018;42(3):725-7.

5. Krause M, Preiss A, Müller G, Madert J, Fehske K, Neumann MV, et al. Intra-articular tibial plateau fracture characteristics according to the "Ten segment classification". Injury. 2016; 47(11):2551-7.

6. Schatzker J, McBroom R, Bruce D. The tibial plateau. The Toronto experience 1968-1975. Clin Orthop 1979; 138:94-104.

7. Kfuri M, Schatzker J. Revisiting the Schatzker classification of tibial plateau fractures. Injury. 2018;49(12): 2252-63.

8. Kaplan PA, Walker CW, Kilcoyne RF, Brown DE, Tusek D, Dussault RG. Occult fracture patterns of the knee associated with anterior cruciate ligament tears: assessment with MR imaging. Radiol. 1992;183(3):835-8.

9. Stallenberg B, Gevenois PA, Sintzoff SA Jr, Matos C, Andrianne Y, Struyven J. Fracture of the posterior aspect of the lateral tibial plateau: radiographic sign of anterior cruciate ligament tear. Radiol. 1993;187 (3):821-5.

10. Menzdorf L, Drenck T, Preiss A, Akoto R, Krause M, Frosch KH. The "Apple- Bite-Fracture": ACL rupture combined with póstero-lateral tibia plateau fracture. 2018; Orthop J Sports Med. 2018; 7(6 suppl 4): 2325967119 S00241.

11. Andrade MAP, Pimenta FS, Silva GMA, Campos TVO, Pires RES. Displaced osteochondral fracture of the posterolateral tibial plateau associated with an acute anterior cruciate ligament injury. Trauma Case Rep. 2019;20:100172.

12. Bowers AL, Huffman GR. Lateral femoral epicondylar osteotomy: an extensile posterolateral knee approach. Clin Orthop Relat Res. 2008;466(7): 1671-7.

13. Kfuri M, Schatzker J, Castiglia MT, Giordano V, Fogagnolo F, Stannard JP. Extended anterolateral approach for complex lateral tibial plateau fractures. J Knee Surg. 2017;30(3):20411.

14. Peccin MS, Ciconelli R, Cohen M. Questionário específico para sintomas 
do joelho "Lysholm knee scoring scale" - Tradução e validação para a língua portuguesa. 2006; 14(5):268-72.

15. Mahmoud TAA, Radwan M. Functional results of percutaneous fixation of displaced tibial plateau fractures assisted by arthroscopy. 2014;49(3):23942.

16. Zhai Q, Luo $\mathrm{C}$, Zhu Y, Yao L, Hu C, Zeng B, et al. Morphological characteristics of split-depression fractures of the lateral tibial plateau (Schatzker type II): a computer tomography-based study. Int Orthop. 2013;37(5):911-7.

17. Chang H, Zheng Z, Shao D, Yu Y, Hou $Z$, Zhang $Y$. Incidence and radiological predictors of concomitant meniscal and cruciate ligament in operative tibial plateau fractures: a prospective diagnostic study. Sci Rep. 2018;8(1): 13317.

18. Durakbasa MO, Kose O, Ermis MN, Demirtas A, Gunday S, Islam C. Measurement of lateral plateau depression and lateral plateau widening in a Schatzker type II fracture can predict a lateral meniscal injury. Knee Surg Sports Traumatol Arthrosc. 2013;21(9):2141-6.

19. Spiro AS, Regier M, Novo de Oliveira A, Vettorazzi E, Hoffmann M, Petersen JP, et al. The degree of articular depression as a predictor of soft-tissue injuries in tibial plateau fracture. Knee Surg Sports Traumatol Arthrosc. 2013;21(3):564-70.

20. Bernholt DL, DePhillipo NN, Grantham WJ, Crawford MD, Aman ZS, Kennedy MI, et al. Morphologic Variants of Posterolateral Tibial Plateau Impaction Fractures in the Setting of Primary Anterior Cruciate Ligament Tear. Am J Sports Med. 2020;48(2): 318-25.

21. Carlson DA. Posterior bicondylar tibial plateau fractures. J Orthop Trauma. 2005; 19(2):73-8.

22. Frosch KH, Balcarek $\mathrm{P}$, Walde $\mathrm{T}$, Stürmer KM. A new posterolateral approach without fibula osteotomy for the treatment of tibial plateau fractures. J Orthop Trauma. 2010;24(8): 515-20.

23. Qiu WJ, Zhan Y, Sun H, Xu YF, Wang YK, Luo C. A posterior reversed Lshaped approach for the tibial plateau fractures - A prospective study of complications (95 cases). Injury. 2015;46(8):1613-8.

24. Cho JW, Kim J, Cho WT, Kim JK, Samal P, Gujjar PH, et al. Approaches and fixation of the posterolateral fracture fragment in tibial plateau fractures: a review with an emphasis on rim plating via modified anterolateral approach. Int Orthop. 2017;41(9): 1887-97.

25. Ackermann C, Frings J, Alm L, Frosch KH. Arthroscopic controlled closed reduction and percutaneous fixation of posterolateral tibia plateau impression fractures. Arthrosc Tech. 2019 19;8(8):e867-74.

26. Lobenhoffer $\mathrm{P}$, Gerich $\mathrm{T}$, Bertram $\mathrm{T}$, Lattermann C, Pohlemann T, Tscheme H. Particular posteromedial and posterolateral approaches for the treatment of tibial head fractures. Unfallchirurg. 1997;100(12):957-67.

27. Solomon LB, Stevenson AW, Baird RP, Pohl AP. Posterolateral transfibular approach to tibial plateau fractures: technique, results, and rationale. $\mathrm{J}$ Orthop Trauma. 2010;24(8):505-14.

28. Pires RES, Giordano V, Wajnsztejn A, Oliveira Santana E Junior, Pesantez $\mathrm{R}$, Lee MA, de Andrade MAP. Complications and outcomes of the transfibular approach for posterolateral fractures of the tibial plateau. Injury. 2016;47(10):2320-5.

29. Shekhar A, Singh A, Laturkar A, Tapasvi S. Anterior Cruciate Ligament Rupture with Medial Collateral Ligament Tear with Lateral Meniscus Posterior Root Tear with Posterolateral Tibia Osteochondral Fracture: A New Injury Tetrad of the Knee. J Orthopaedic Case Rep. 2020;10(3):36-42.

Recebido em: 20/09/2020

Aceito para publicação: 19/12/2020

Conflito de interesse: não

\section{Endereço para correspondência:}

Robinson Esteves Pires

E-mail: robinsonestevespires@gmail.com 